\title{
Expansión del proyecto de idiomas de la Sede del Pacífico de la UCR: aciertos y desafíos
}

\section{Expansion of a Language Project at the UCR Pacific Campus: Correct Actions and Challenges}

\author{
Rosberly López Montero \\ Universidad de Costa Rica \\ Puntarenas, Costa Rica \\ ros_ber1y@yahoo.es \\ Kerry Navarrete Padilla \\ Universidad de Costa Rica \\ Puntarenas, Costa Rica \\ kerry.navarrete@hotmail.com
}

Recibido: 17/04/2020 Aceptado:10/08/20

Resumen. El presente artículo pretende analizar el impacto que ha tenido la expansión hacia comunidades fuera de la sede universitaria, de parte del proyecto de extensión docente ED-2884 "Idiomas para la Comunicación Internacional" de la UCR, Sede del Pacífico, mediante la descripción de sus aciertos y desafíos más importantes en este proceso. El mismo revela un crecimiento en el impacto del proyecto en la región, facilitando el acceso al aprendizaje de idiomas a una población mayor; pero, a la vez, detalla los desafíos que debe enfrentar para mantener una oferta de cursos de calidad vigente en dichas comunidades.

Palabras clave: extensión universitaria, idiomas, multilingüismo, regionalización.

Abstract. This paper analyzes the impact of the outreach Language project ED-2884 at the Pacific Regional Center of Universidad de Costa Rica in terms of its extension to communities surrounding the university campus. It describes the most relevant correct actions and challenges faced during this process. The paper reveals an increase in the project's impact in the region, letting a bigger population access language learning. At the same time, it details the challenges the project must face to keep a quality offer of courses available in such communities.

Keywords: languages, multilingualism, regionalization, university extension. 


\section{Introducción}

El objetivo principal del proyecto de idiomas de la Sede del Pacífico de la Universidad de Costa Rica (ED-2884) es brindar capacitación en distintos idiomas a la población puntarenense sin necesidad de dejar su provincia; para lograrlo se trabaja de manera transversal en el aprendizaje colaborativo, la internacionalización y los valores, como la solidaridad y el respeto, pues el proyecto tiene la convicción de que, más allá de un aspecto relacionado con la globalización y la economía, aprender otras lenguas contribuye a la reducción de estereotipos y a forjar sociedades más justas y solidarias, de esto hay prueba en el trabajo presentado por Navarrete (2018), en el que se desarrolla una indagación de estudiantes de tres distintos idiomas, los cuales afirman haber reducido sus estereotipos de la cultura meta al haber estudiado el idioma.

Asimismo, a lo largo del año 2018 y en el marco del Año de la Regionalización Universitaria, el proyecto extendió su servicio a comunidades fuera de la Sede, impactando distintas localidades de la Región Pacífico Central, como Cóbano, Miramar, Orotina, y Esparza. Así también, se ha expandido hasta adentrarse en distintas empresas de la zona, impactando distintos tipos de poblaciones, por lo que el presente documento tiene el propósito de hacer referencia a los aciertos y desafíos presentados al expandir la enseñanza de idiomas de un proyecto de extensión docente a comunidades fuera de la sede universitaria.

\section{Antecedentes}

El proyecto de idiomas de la Sede del Pacífico de la Universidad de Costa Rica: ED-2884 "Idiomas para la Comunicación Internacional" imparte su primer grupo de cursos a partir de enero de 2012. Desde entonces, la Sede ha recibido con sus puertas abiertas a estudiantes de diferentes sectores de la comunidad puntarenense y de zonas vecinas. Mayoritariamente, los cantones Central y de Esparza han sido los más beneficiados, pues de ahí provienen la mayor cantidad de estudiantes que toman los cursos (López, 2014); sin embargo, no han sido los únicos, ya que vecinos de zonas como Orotina, Miramar, Cóbano, Chomes y Quebrada Ganado, entre otras, se desplazan cada semana a tomar las lecciones. Adicionalmente, vecinos de lugares un poco más alejados, pero que residen en Puntarenas en un período corto por estudio o trabajo, también se benefician al llevar estas capacitaciones.

Desde sus inicios, este proyecto autofinanciado ha impartido más de cuatrocientos cursos en las distintas comunidades, beneficiando a más de cien personas por período (López, 2017); este número ha aumentado significativamente desde su expansión a otras comunidades. 
URL: https://www.revistas.una.ac.cr/index.php/dialogo/index

CoRreo electrónico: universidadendialogo@una.ac.cr

DOI: https://doi.org/10.15359/udre.10-2.2

\section{Metodología del proyecto}

El proyecto se desarrolla en cuatro períodos lectivos al año, de diez semanas cada uno, con dos semanas de receso entre cada período, el cual corresponde a procesos de matrícula en línea. Para el desarrollo de las clases y los programas de curso se toman en consideración las necesidades específicas de la población a atender, además de la cooperación y la internacionalización como ejes transversales en la metodología de los cursos, esto con el fin de lograr sociedades más justas mediante el desarrollo de actitudes positivas ante otras lenguas y culturas; por supuesto, dentro de un enfoque de comunicación.

De igual manera, se trabaja en la investigación para generar conocimiento que pueda ser aplicado en el aula, de modo que enriquezca la docencia y los métodos de trabajo de cada docente. Además, con el mismo objetivo, se desarrollan capacitaciones para los y las docentes del proyecto, dependiendo de las necesidades que se presenten en el mismo, por ejemplo, algunos de los temas en los que se ha brindado formación son mediación pedagógica en la adquisición de idiomas extranjeros para poblaciones específicas: niños y niñas, adolescentes, personas adultas, desarrollo de diferentes habilidades de idiomas extranjeros, temas de inclusión, concientización de estudiantes con discapacidades, y recientemente se implementó un sistema de capacitaciones abierto para docentes de Inglés de la zona, estas se imparten tres veces al año.

Para dar seguimiento a procesos de calidad de los cursos, se realizan monitoreos mediante censos aplicados a la población estudiantil, sobre su opinión con respecto a los contenidos enseñados, al personal docente, a las instalaciones y al trabajo desde la coordinación del proyecto. Estos datos son sistematizados a través de las notas publicadas en el portal de Acción Social de la Universidad de Costa Rica y en publicaciones de revistas académicas; este modo de sistematizar los resultados y las opiniones de la población participante ha resultado muy provechosa para el proyecto, pues permite documentar y, por lo tanto, contar con fuentes oficiales que detallen lo realizado por el proyecto, lo cual a su vez se divulga en distintos espacios. Así, la información no se queda en un informe anual, sino que está al alcance de la comunidad en general sin espacios temporales restringidos.

Cabe destacar que a partir del 2018 el proyecto adopta un sistema de matrícula en línea facilitado por la Fundación UCR, lo cual permite que las personas se inscriban sin tener que desplazarse a la sede universitaria; esto facilita el proceso de matrícula en otras comunidades. Ahora bien, para determinar las nuevas sedes en las que se ofrecerán cursos como parte del proyecto, por lo 
general, se cuenta con conocimiento de la necesidad de los cursos en la zona, ya sea por medio de informes de las necesidades de las comunidades que emite la coordinación de Acción Social de la Sede o porque algún habitante de la zona haga una petición al proyecto mediante redes sociales, o se cuenta con algún contacto que hace la sugerencia y contribuye con las gestiones para contactar el local donde se impartirán las lecciones; los mismos suelen ser parte de asociaciones comunales, del gobierno local e incluso estudiantes que tomaron los cursos en las instalaciones de la Sede universitaria y ahora cambiaron su residencia.

Entre las comunidades a las que se ha expandido el proyecto están: Cóbano, Orotina y Esparza, en estas comunidades se han ofrecido cursos para niños y niñas desde los cinco años de edad y para personas jóvenes y adultas. Además, en Miramar se ofrecen cursos para la población infantil a partir de los cinco años de edad. Por otra parte, se trabaja con poblaciones específicas de empresas ubicadas en El Roble y en Esparza, lo cual brinda a dichos trabajadores oportunidades de crecimiento profesional en el mismo lugar de trabajo, con horarios que se adecúan a sus necesidades.

\section{Desarrollo del tema}

\section{Regionalización universitaria y la extensión docente}

Tal como lo expresa la Asociación de Estudiantes de Occidente en su presentación en el VII Congreso Universitario (2014), el proceso de regionalización de la Universidad de Costa Rica inició en 1960 en medio de una discusión sobre la creciente necesidad de ofrecer oportunidades de educación superior a la población joven de zonas rurales, para así promover el desarrollo económico en el país, por lo que se hace necesario democratizar la universidad pública. Este hecho obtiene el apoyo del Consejo Universitario en la sesión $\mathrm{N}^{\circ} 1571$ de 1967, lo que consolida la apertura de la Sede Regional de Occidente en 1968 (pp. 2-3). Es así como sucesivamente se inicia con la apertura de otras sedes regionales en diferentes zonas del país, lo cual beneficia a las comunidades, brindando mayor acceso a la educación superior pública para las y los jóvenes que viven fuera del área metropolitana, pero también se fortalece el vínculo de la universidad con las comunidades, al promover desarrollo social y económico por medio de sus proyectos de extensión docente, extensión cultural y los trabajos comunales universitarios que se gestionan desde la coordinación de Acción Social de cada sede. 
URL: https://www.revistas.una.ac.cr/index.php/dialogo/index

CORREO ELECTRÓNICO: universidadendialogo@una.ac.cr

DOI: https://doi.org/10.15359/udre.10-2.2

En ese sentido, cabe mencionar el surgimiento de la acción social en la Universidad de Costa Rica. De acuerdo con la Vicerrectoría de Acción Social (2015), en el III Congreso Universitario llevado acabo entre 1971-1972 se discutió sobre el quehacer académico de la Universidad de Costa Rica, y es así como en el Estatuto Orgánico de 1974 surge la acción social como un área sustantiva de la educación superior pública en la Universidad de Costa Rica, por lo que se comienza con dicha Vicerrectoría de la mano de doña María Eugenia Dengo, educadora y primera vicerrectora de Acción Social de la Universidad de Costa Rica entre los años 1974-1976.

De acuerdo con la Universidad de Costa Rica (2016), el propósito de la Vicerrectoría de Acción Social es facilitar el vínculo entre la Universidad de Costa Rica y la sociedad, para que "(...) la institución pueda cumplir efectivamente con el propósito de contribuir con las transformaciones que se necesitan para el logro del bien común" (p. 23).

Para ello, dicha Vicerrectoría posee una serie de principios, entre los cuales se destacan el humanismo, el diálogo de saberes, los enfoques de derechos, la inclusión, la participación, la equidad, la cooperación, entre otros, de los cuales hace uso en sus procesos de aprendizaje, contribuyendo así con el pleno desarrollo de las capacidades humanas, y creando una sociedad justa, inclusiva, participativa, respetuosa de los derechos humanos, por lo cual es necesario que llegue a más personas y comunidades vulnerables por medio de las sedes regionales y así cumpla su objetivo.

Además, en cuanto a los esfuerzos por la regionalización para la educación superior pública, es importante mencionar el compromiso de cuatro universidades públicas del país que, en el año 1998 y desde el Consejo Nacional de Rectores (Conare), firmaron el Convenio Marco para el Desarrollo de las Sedes Regionales Interuniversitarias en Educación Superior Universitaria Estatal de Costa Rica, entre la Universidad de Costa Rica (UCR), el Instituto Tecnológico de Costa Rica (ITCR), la Universidad Nacional (UNA) y la Universidad Estatal a Distancia (UNED). De acuerdo con el Conare (1998), este convenio se basa en políticas institucionales sobre regionalización y desconcentración para ayudar al desarrollo integral de las diferentes zonas del país, además de buscar que las sedes regionales ofrezcan opciones académicas que respondan a las necesidades que tienen las comunidades donde se encuentran sus sedes. Estos esfuerzos hechos desde las universidades públicas muestran el compromiso de las mismas por ser accesibles a la mayor cantidad de personas posibles y tener presencia en diferentes zonas del país. 


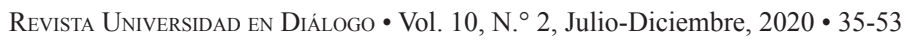

ISSN 2215-2849 • EISSN: 2215-4752

URL: https://www.revistas.una.ac.cr/index.php/dialogo/index Correo electrónico: universidadendialogo@una.ac.cr

DOI: https://doi.org/10.15359/udre.10-2.2

No obstante, son evidentes las grandes divergencias económico-sociales entre la Gran Área Metropolitana y las zonas periféricas, además de la creciente demanda de diversificación en la oferta académica, dichas diferencias han ocasionado una condición de estancamiento en cuanto a la cobertura en términos de acceso a la educación y de servicios ofrecidos por parte de las casas de enseñanza superior hacia la población universitaria en general y las regiones periféricas donde ella se encuentra.

Ahora bien, si este es el panorama para el estudiantado con los requisitos para ingresar a una vida universitaria, la universidad pública se hace aún más lejana para aquellas personas que no tienen las mismas oportunidades de ingreso, por lo que opciones de preparación académica adicional en las zonas periféricas, inclusive en aquellas en las que no existan sedes universitarias, es parte de la labor de la contribución de la acción social. Por eso, la existencia de proyectos de acción social que expandan sus servicios a comunidades fuera de las sedes universitarias contribuye a cumplir con el compromiso de democratización de la educación superior, el cual es la idea original de la regionalización universitaria.

\section{Expansión del proyecto ED-2884 "Idiomas para la Comunicación Internacional"}

Debido al compromiso que tiene la coordinación de Acción Social de la Universidad de Costa Rica, en la Sede del Pacífico se gestionan diferentes proyectos de extensión docente, entre los que se encuentra el Proyecto ED2284 "Idiomas para la Comunicación Internacional", el cual tiene como objetivo principal brindar capacitación en distintos idiomas a la población puntarenense sin necesidad de dejar su provincia. Esto ha permitido que las comunidades ubicadas alrededor de dicha sede se vean beneficiadas con cursos de idiomas de buena calidad y a bajos costos, y a la vez la Universidad de Costa Rica contribuye con el desarrollo socioeducativo de las personas locales, lo cual es un pilar de la Institución.

Lo anterior, a su vez, mejora la calidad de vida de la población, y así se contribuye con la reducción de la brecha socioeconómica entre las zonas centrales y las regiones rurales y costeras del país, pues es innegable que el dominio de lenguas extranjeras es un requisito indispensable en muchas ofertas laborales y académicas, y como lo afirma Hasbún (2007), “(...) cuando a un individuo se le niega el acceso a las lenguas extranjeras, implícitamente, se le están limitando las posibilidades de mejorar su posición socioeconómica" (p. 111). 
URL: https://www.revistas.una.ac.cr/index.php/dialogo/index

CORREO ELECTRÓNICO: universidadendialogo@una.ac.cr

DOI: https://doi.org/10.15359/udre.10-2.2

Además, cabe mencionar que Acuña, Barahona y Ceciliano (2010) manifiestan que "la oferta pública debe considerar adecuados niveles de descentralización de los servicios educativos en idiomas, que ofrezcan el aprendizaje con criterios de calidad y eficiencia como se ofrecen en las regiones centrales del país" (p. 17). Basados en los autores mencionados anteriormente y otros, además del compromiso que tiene la Universidad de Costa Rica con la acción social en las comunidades, es que la expansión del proyecto de idiomas ED-2884 a otras comunidades responde al cumplimiento de su objetivo específico: expandir la capacitación en idiomas a otras comunidades e instituciones fuera de la Sede del Pacífico según necesidades identificadas en la población. Para el cumplimiento de este objetivo, se establecen las siguientes metas:

A. Meta 1: Impartir cursos fuera de las instalaciones de la Sede del Pacífico, tomando como indicadores el número de idiomas y cursos impartidos

B. Meta 2: Adaptar los programas curriculares de modo que correspondan con las necesidades identificadas en estas poblaciones.

Los indicadores del alcance de estas metas se centran en el número de programas desarrollados y el contar con un programa de reducción de costos para beneficiar a la población estudiantil de recursos económicos limitados; de este modo, se alcanza otro tipo de población que usualmente no tendría posibilidades de tomar estos cursos.

El proceso del cumplimiento del objetivo específico, considerando las metas alcanzadas y las acciones llevadas a cabo, se describe a continuación:

Sin duda, en el año 2018, el proyecto concretó uno de sus logros más importantes: su expansión a otras comunidades fuera de las instalaciones universitarias en el Cocal. La primera comunidad hermana del proyecto en la que se desarrollaron los cursos fue en el distrito de Cóbano, ubicado en el extremo sur de la Península de Nicoya. Se inicia con la apertura de cinco grupos de inglés en su primer período: dos grupos que forman parte del programa de inglés para jóvenes y personas adultas, un grupo del programa de inglés para preescolares, dirigido a la población infantil entre los cinco y seis años de edad, dos grupos del programa de inglés para escolares, uno de ellos dirigido a niños y niñas desde los siete hasta los nueve años de edad, y el grupo restante para niñas y niños de diez a doce años. 
Revista Universidad en DiÁlogo • Vol. 10, N. ํ 2, Julio-Diciembre, 2020 • 35-53

ISSN 2215-2849 • EISSN: 2215-4752

URL: https://www.revistas.una.ac.cr/index.php/dialogo/index Correo eleCtrónico: universidadendialogo@una.ac.cr

DOI: https://doi.org/10.15359/udre.10-2.2

Para la apertura de dichos cursos se contacta a la Asociación de Desarrollo Integral de Cóbano, cuyo papel fue clave, ya que debido a un convenio entre esta y la Universidad de Costa Rica, Sede del Pacífico, se logra el préstamo de la Casa de la Cultura para impartir las lecciones. Además, es importante mencionar que los miembros de la Asociación contribuyeron de gran forma al brindar publicidad sobre la apertura de los cursos en sus redes sociales y con la colocación de afiches en distintos negocios de la comunidad, y finalmente en el período de matrícula facilitaron espacios en sus propios negocios para hacer uso del Internet y de equipo de cómputo para capacitar a la población en el proceso de la matrícula.

La segunda comunidad donde el proyecto de idiomas tiene presencia es en Miramar, en este caso se promocionó la apertura de los cursos de Inglés para niñas y niños debido a que padres y madres de familia contactaron al proyecto solicitando la apertura de cursos de Inglés, pues les era muy difícil desplazarse hasta las instalaciones de la Sede en el Cocal. Así es como la misma familia, por el gran interés, y debido a las buenas referencias hechas por un grupo de madres y padres de la comunidad de Montes de Oro, que ya habían matriculado a sus hijos e hijas en los cursos, deciden ayudar al proyecto con la búsqueda de un local para impartir las lecciones; una vez que las familias brindan recomendaciones de lugares, el proyecto contacta a las juntas educativas de las escuelas de la comunidad, con las que sostiene reuniones y se establecen convenios para facilitar las instalaciones de las escuelas, una fue la Escuela José María Zeledón y la otra la Escuela de Santa Rosa, ambas ubicadas en el cantón de Montes de Oro. Se ha logrado abrir dos grupos del programa de inglés para niños y niñas, uno dirigido a población entre los siete y los nueve años de edad, y el otro para población de cinco y seis años de edad.

La tercera comunidad a donde se expande el proyecto de idiomas es Orotina, Alajuela. Esta comunidad es escogida por medio de las redes sociales del proyecto, y algunos estudiantes que viajaban de dicha comunidad a la Sede de la Universidad en El Cocal, Puntarenas, a tomar los cursos hicieron la solicitud, así que en el segundo período del año 2018 se inicia en Orotina con tres grupos: uno de ellos del programa de inglés para jóvenes y personas adultas, y dos grupos con el programa de inglés para escolares, uno para niños y niñas de los siete a los nueve años de edad, y el tercer grupo dirigido a niños y niñas entre los diez y los doce años de edad. En esta comunidad, la vinculación entre el proyecto y la Municipalidad de Orotina fue muy importante, ya que se creó un convenio con esta para que facilitara el Centro de Capacitaciones durante los períodos de matrícula, y para impartir las clases. Además, la Municipalidad de Orotina contribuyó con la divulgación de la apertura del proyecto mediante sus redes sociales oficiales. 
URL: https://www.revistas.una.ac.cr/index.php/dialogo/index

CORREO ELECTRÓNICO: universidadendialogo@una.ac.cr

DOI: https://doi.org/10.15359/udre.10-2.2

Finalmente, la cuarta comunidad es Nances, Esparza. Debido a la experiencia en Miramar de Montes de Oro, y aprovechando que la Sede cuenta con un recinto en dicha comunidad, desde la coordinación del proyecto se decide habilitar los cursos en las instalaciones de la sede en Esparza. En dicha comunidad se ha logrado la apertura de cuatro grupos, uno de ellos perteneciente al programa de Lenguaje de Señas Costarricense (Lesco), el cual es dirigido a personas jóvenes y adultas, y los tres restantes del programa de inglés para la población infantil, dos de ellos para niñas y niños entre los diez y los doce años de edad, y uno para niñas y niños de siete a nueve años de edad.

Además, se impartieron capacitaciones en dos centros de trabajo con el programa denominado Inglés en su Empresa, facilitando esta opción a personas trabajadoras a las que se les dificulta tomar este tipo de cursos por cuestiones de horario laboral, pero, al ofrecer el curso directamente a los colaboradores de las empresas, se coordinó con los departamentos de recursos humanos para adaptar los horarios según lo solicitado por estos departamentos, y así ellos adquirían el compromiso de brindar ese espacio a sus colaboradores para que tomaran el curso. Así las cosas, durante el año 2018 el proyecto se desarrolló en los siguientes lugares:

Para población en general:

- Sede del Pacífico, El Cocal de Puntarenas

- Cóbano, Puntarenas

- Miramar, Puntarenas

- Sede del Pacífico, Esparza

- Orotina, Alajuela

Para empresas:

- Hotel Double Tree by Hilton (posteriormente Hotel Fiesta Resort), en el Roble de Puntarenas

- Austin Powder, en Paraíso de Esparza

El ejemplo más claro del alcance de la segunda meta es el programa Inglés en su Empresa, el cual, como ya se dijo, se ha adecuado para que los empleados de distintas empresas se capaciten en la lengua inglesa, adaptándonos a sus horarios e instalaciones; para esto se solicita que dichas instalaciones cuenten 
Revista Universidad en Diálogo • Vol. 10, N. ํ 2, Julio-Diciembre, 2020 • 35-53

ISSN 2215-2849 • EISSN: 2215-4752

URL: https://www.revistas.una.ac.cr/index.php/dialogo/index Correo electrónico: universidadendialogo@una.ac.cr

DOI: https://doi.org/10.15359/udre.10-2.2

con los recursos mínimos para impartir lecciones y de este modo evitar la afectación en la calidad de los cursos. Además, en el caso del Hotel Double Tree by Hilton se adaptaron algunos contenidos de cada módulo en cuanto a vocabulario, para que se relacionaran con el departamento en el que los estudiantes se desempeñan laboralmente.

Es importante mencionar que, en el caso de empresas, se trata de un cuerpo de colaboradores en muchas ocasiones de bajos recursos y que difícilmente podrían tomar esas capacitaciones por sí solos, no solamente por un asunto económico, sino por un asunto de tiempo, ya que las agendas de sus trabajos dificultan que se integren a los horarios de los cursos, por lo cual llevarlos hasta sus trabajos, ajustándose a sus horarios laborales, es de gran apoyo para su mejoramiento profesional.

De modo similar, sucede con el programa general de inglés para jóvenes y personas adultas, el cual se imparte en la Sede de El Cocal en módulos de setenta horas, hay lugares, como Cóbano, en que, por cuestiones de servicio de transporte público, a las personas se les dificulta trasladarse más de una vez por semana, por lo que el programa se adecuó de tal forma que estuviese dividido en una mayor cantidad de módulos (un módulo más), pero menor cantidad de horas por módulo para que fuese solamente una sesión semanal, lo mismo sucede con el programa de Inglés en su Empresa, se adecua el número de horas de cada módulo al tiempo que tengan disponibles los colaboradores de las empresas.

No menos importante es mencionar que la expansión a otros sectores incluye parte de la población que no puede costearse los cursos, por lo que se inició la implementación del Plan de Reducción de Costos para Becados UCR; con dicho plan es posible utilizar el sistema de becas universitario para determinar un porcentaje de reducción en el precio de los cursos de Inglés (por ser el idioma con mayor demanda) para los y las estudiantes que posean beca, lo que les facilita el acceso a dichos cursos. De modo que, estudiantes con beca 1,2 y 3 (que obtienen una exoneración del $45 \%$ al $90 \%$ en sus estudios) obtienen un $50 \%$ de descuento en el precio del curso, mientras que los becados con categoría 4 y 5 (100\% de exoneración), un $90 \%$. De modo que, en este año, quince estudiantes de distintas carreras, en su mayoría beca 5, se han acogido a este plan, lo que equivale a aproximadamente el financiamiento de unos $\mathbb{C} 2000000$ por parte del proyecto. Sin embargo, lo realmente importante de recalcar en este punto es la posibilidad que se les ha abierto a estudiantes universitarios de acceder a capacitación en el área de idiomas, mientras estudian para sacar su título universitario. 
URL: https://www.revistas.una.ac.cr/index.php/dialogo/index

CORREO ELECTRÓNICO: universidadendialogo@una.ac.cr

DOI: https://doi.org/10.15359/udre.10-2.2

\section{Aciertos}

Es importante recalcar que durante el año 2018 el proyecto de idiomas tuvo un aumento considerable en el número de estudiantes matriculados por período, y se puede asegurar que el logro principal en este año sin duda tiene que ver con la expansión del proyecto a otras comunidades. Esto permitió un aumento significativo en la población estudiantil, lo que a su vez indica que mayor cantidad de personas se vieron beneficiadas con estos cursos.

En resumen, durante el primer período del 2018, se abrieron 24 grupos de diferentes programas (jóvenes y personas adultas, niños y niñas en edades escolares y preescolares) e idiomas, predominando el inglés, para un total de 278 estudiantes. Durante el segundo período del mismo año se tuvo la apertura de 34 grupos, en los cuales se matricularon 385 estudiantes, en el tercer período del año 2018 se logra abrir 29 grupos, con un total de 328 estudiantes registrados, y para el cuarto y último período del año 2018 se trabajó con 25 grupos, con un total de 233 estudiantes.

El siguiente gráfico muestra el comportamiento de las matrículas durante los cuatro períodos del año 2018 con su respectiva sede.

Figura 1. Número de estudiantes por período y sede durante el año 2018

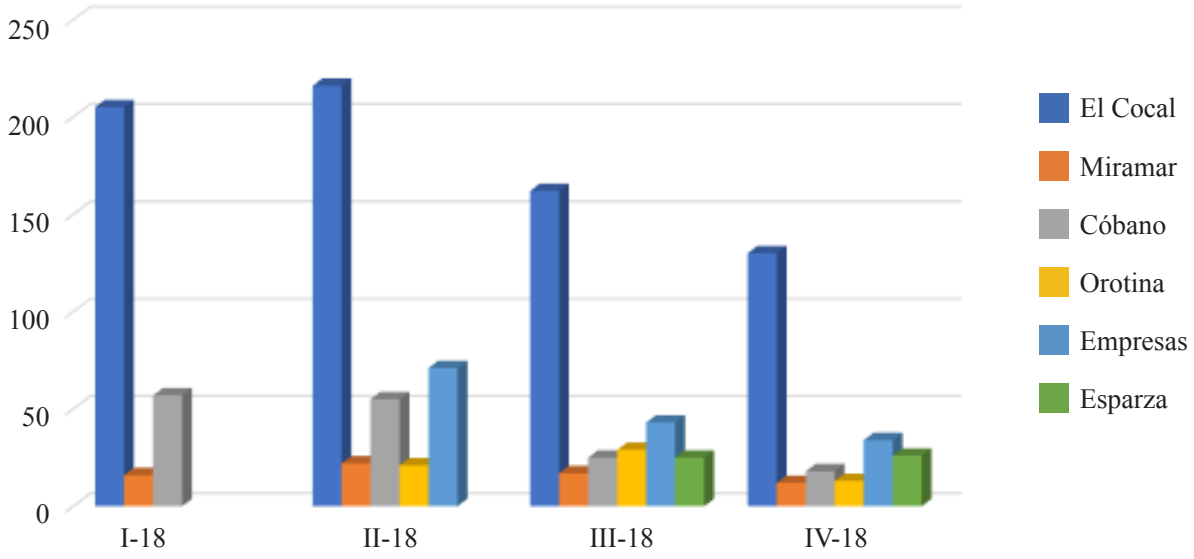

Nota: Elaboración propia con datos del Proyecto ED-2284 "Idiomas para la Comunicación Internacional”. 
Como se aprecia en la figura 1, la comunidad de El Cocal mantuvo la mayor concentración de estudiantes; no obstante, es de suma importancia para este proyecto el número de estudiantes matriculados en las demás sedes, pues representa el inicio de la expansión del proyecto y el cumplimiento de un objetivo específico del mismo. A la vez, incorpora poblaciones que años anteriores no tenían la oportunidad de acceder a los cursos de idiomas ofrecidos por la Universidad de Costa Rica, Sede del Pacífico, a través del Proyecto de Extensión Docente ED-2284 "Idiomas para la Comunicación Internacional", el cual tiene la proyección de aumentar dicha matrícula conforme se vaya consolidando el proyecto, al pasar del tiempo en dichas comunidades, como ha sucedido en la comunidad de El Cocal.

Los siguientes cuadros muestran con mayor claridad el número exacto de estudiantes matriculados en cada período y sede.

Tabla 1

Estudiantes matriculados el primer período, distribuido por sede

\begin{tabular}{lc}
\hline Comunidad & Población estudiantil \\
\hline El Cocal & 205 \\
Cóbano & 57 \\
Miramar & 16 \\
Tres sedes & 278 estudiantes
\end{tabular}

Nota: Elaboración propia con datos del Proyecto ED-2284 "Idiomas para la Comunicación Internacional".

Como se aprecia en la tabla 1, en la comunidad de El Cocal se encontraron inscritos 205 estudiantes del total matriculado durante el primer período del año 2018, que comprende los meses de enero hasta mediados de abril; esto obedece a la mayor demanda por parte de la población de otros idiomas, diferentes al inglés, y del programa de inglés para la población infantil de diferentes edades. No obstante, en este período se inicia con la primera comunidad de la expansión, la cual es Cóbano, y con un grupo de niños y niñas en Miramar. 
URL: https://www.revistas.una.ac.cr/index.php/dialogo/index

CORREO ELECTRÓNICO: universidadendialogo@una.ac.cr

DOI: https://doi.org/10.15359/udre.10-2.2

Tabla 2

Estudiantes matriculados el segundo período, distribuido por sede

\begin{tabular}{|c|c|}
\hline Comunidad & Población estudiantil \\
\hline El Cocal & 216 \\
\hline Inglés en su Empresa (Hotel Double Tree by Hilton) & 71 \\
\hline Cóbano & 55 \\
\hline Miramar & 22 \\
\hline Orotina & 21 \\
\hline Cinco sedes & 385 estudiantes \\
\hline
\end{tabular}

Nota: Elaboración propia con datos del Proyecto ED-2284 "Idiomas para la Comunicación Internacional".

La tabla 2 resume la situación de las matrículas durante el segundo período, que abarca los meses de finales de abril a junio, como se puede observar, la sede de El Cocal sigue predominando en el número de estudiantes matriculados, con un total de 216, seguidamente se inicia con el programa de Inglés en su Empresa, desarrollado en el Hotel Double Tree by Hilton, ubicado en El Roble, Puntarenas, con un total de 71 estudiantes, quienes son empleados del hotel, y fueron distribuidos en grupos dependiendo del área donde se desempeñaban laboralmente, con el fin de ofrecerles un curso adecuado a las necesidades de cada departamento; se mantiene la sede de Cóbano y Miramar, y se incorpora la sede de Orotina con un total de 21 estudiantes.

Además, cabe mencionar que algunos cursos, por no alcanzar el cupo mínimo, fueron cerrados, y en estos se encontraban un total de 18 personas, a quienes lamentablemente se les comunicó que el curso que matricularon no sería abierto; es decir, en total fueron 403 personas las que acudieron al proyecto y realizaron su matrícula. 
Revista Universidad en Diálogo • Vol. 10, N. ํ 2, Julio-Diciembre, 2020 • 35-53

ISSN 2215-2849 • EISSN: 2215-4752

URL: https://www.revistas.una.ac.cr/index.php/dialogo/index CORREO ElECTRÓNICO: universidadendialogo@una.ac.cr

DOI: https://doi.org/10.15359/udre.10-2.2

Tabla 3

Estudiantes matriculados el tercer período, distribuido por sede

\begin{tabular}{|c|c|}
\hline Comunidad & Población estudiantil \\
\hline El Cocal & 162 \\
\hline Inglés en su Empresa (Hotel Double Tree by Hilton) & 52 \\
\hline Esparza & 29 \\
\hline Cóbano & 25 \\
\hline Orotina & 25 \\
\hline Inglés en su Empresa (Austin Powder) & 18 \\
\hline Miramar & 17 \\
\hline Siete sedes & 328 estudiantes \\
\hline $\begin{array}{l}\text { Nota: Elaboración propia con datos del Proyecto } \\
\text { Comunicación Internacional". }\end{array}$ & -2284 “Idiomas para la \\
\hline \multicolumn{2}{|c|}{$\begin{array}{l}\text { En la tabla } 3 \text { se muestra que ya para el tercer período del año } 2018 \text {, que cubre } \\
\text { los meses de julio a setiembre, el proyecto de idiomas cuenta con siete sedes } \\
\text { pues se incorpora la sede en Nances, Esparza, con un total de } 29 \text { estudiantes, y } \\
\text { otra sede del programa Inglés en su Empresa, en esta ocasión se desarrolla con } \\
\text { los colaboradores de la empresa Austin Powder, ubicada en Paraíso, Esparza, y } \\
\text { en dicha ocasión se atiende una población de } 18 \text { estudiantes, quienes trabajar } \\
\text { en la empresa mencionada anteriormente. Pero además de estas sedes, cabe } \\
\text { mencionar que se mantienen las cinco de los períodos anteriores. }\end{array}$} \\
\hline \multicolumn{2}{|c|}{$\begin{array}{l}\text { Tabla } 4 \\
\text { Estudiantes matriculados el cuarto período, distribuido por sede }\end{array}$} \\
\hline Comunidad & Población estudiantil \\
\hline El Cocal & 130 \\
\hline $\begin{array}{l}\text { Inglés en su Empresa (Hotel Fiesta Resort antiguo } \\
\text { Double Tree by Hilton) }\end{array}$ & 34 \\
\hline Esparza & 26 \\
\hline Cóbano & 18 \\
\hline Orotina & 13 \\
\hline Miramar & 12 \\
\hline Siete sedes & 233 estudiantes \\
\hline
\end{tabular}

Nota: Elaboración propia con datos del Proyecto ED-2284 "Idiomas para la Comunicación Internacional" 
URL: https://www.revistas.una.ac.cr/index.php/dialogo/index

CORREO ELECTRÓNICO: universidadendialogo@una.ac.cr

DOI: https://doi.org/10.15359/udre.10-2.2

En la tabla anterior se puede percibir que, para el cuarto período del año 2018, desarrollado en los meses de octubre a la primera quincena de diciembre, se mantuvieron seis sedes activas, la que no continuó en este período es la sede de la empresa Austin Powder, debido a las demandas laborales propias de la época. No obstante, durante todo el año 2018 se mantuvieron activas las demás sedes de la expansión, con diferentes retos que serán analizados más adelante, pero el servicio académico por medio de los cursos de extensión docente se siguió brindando a las comunidades.

Como es evidente en los cuadros y gráfico anteriores, hay diferencias sobre todo en el número de estudiantes matriculados en la sede de El Cocal, y las demás sedes, propias de la expansión del proyecto, por ello se presenta el siguiente gráfico para ejemplificar lo que sucede en la Sede base del proyecto, la cual es en la comunidad de El Cocal, Puntarenas, y con el resto de sedes de la expansión.

Figura 2. Situación del proyecto en la Sede de El Cocal vs las sedes de la expansión

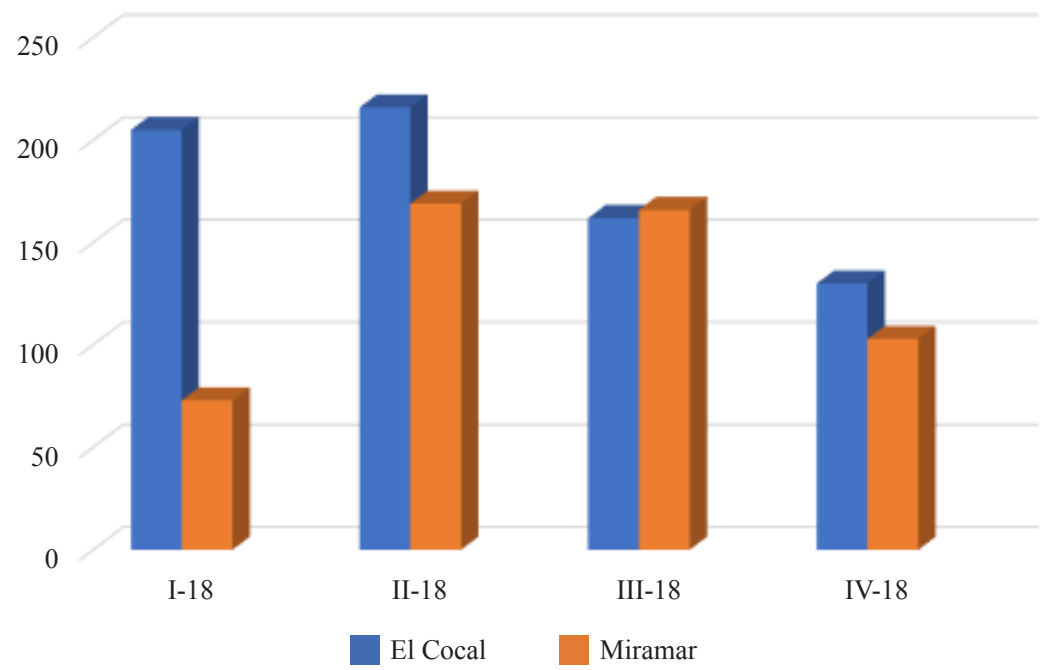

Nota: Elaboración propia con datos del Proyecto ED-2284 "Idiomas para la Comunicación Internacional".

En la figura 2 se muestran las diferencias de matrícula del proyecto de idiomas entre la Sede de la Universidad de Costa Rica en la comunidad de El Cocal, Puntarenas, y las comunidades donde se llevó la expansión del proyecto, si 
bien es cierto que, al verse las comunidades de la expansión por separado, la diferencia de matrícula con respecto a la Sede de El Cocal es significativa, al articularlas se puede observar que las diferencias en términos de cantidad de estudiantes no es tan distante, salvo durante el primer período del año 2018, pues en ese momento se estaba iniciando y explorando la expansión del proyecto a otras comunidades, no obstante, como se puede observar en la figura 6 , durante el tercer ciclo hubo un mayor número de estudiantes matriculados en comunidades fuera de la Sede de la Universidad de Costa Rica, en El Cocal, Puntarenas. En otras palabras, el proyecto ha mantenido un balance con respecto al compromiso de su objetivo específico de expandir el proyecto a comunidades fuera de las instalaciones de la Universidad de Costa Rica, y mantener en crecimiento el proyecto dentro de sus propias instalaciones, lo cual se traduce en mayores oportunidades para las comunidades de acceder a cursos de idiomas en sus propios lugares de residencia.

\section{Desafíos}

Si bien es cierto, como se expuso anteriormente, el proyecto de idiomas ha logrado una de sus metas, la cual es impartir cursos fuera de las instalaciones de la Universidad de Costa Rica, Sede del Pacífico, el mismo se enfrenta con algunos desafíos, los cuales, desde la coordinación del proyecto, se han venido trabajando para no afectar el desempeño y la calidad de los cursos ofrecidos.

Uno de esos desafíos es lograr que los y las docentes sean de la zona donde se imparten los cursos, ya que esto brinda algunas ventajas, como que la persona docente conoce la población meta, las particularidades de la comunidad y asegura un mayor compromiso, pues, al ser las comunidades un poco alejadas de zonas centrales, pueden existir dificultades de acceso, las cuales se reducen si la persona docente es de la localidad. Además, el proyecto fija una serie de requisitos que deben cumplir los y las docentes para asegurar la calidad de los cursos, y por lo tanto desde la coordinación del proyecto se realiza una selección cuidadosa. Para ello, se hace una publicación de la necesidad de docentes para impartir cursos en las comunidades, junto con los respectivos requisitos, se reciben los currículums por un tiempo definido, se hace la revisión de los mismos y se seleccionan aquellos que cumplen con lo solicitado, seguidamente se convoca a una entrevista para conocer más detalles de la persona docente que no pueden ser reflejados en su hoja de vida. Finalmente, se les ofrece la oportunidad laboral a aquellos docentes que se consideran aptos para aportar y seguir las metodologías pedagógicas que mantiene el proyecto. 
URL: https://www.revistas.una.ac.cr/index.php/dialogo/index

CORREO ELECTRÓNICO: universidadendialogo@una.ac.cr

DOI: https://doi.org/10.15359/udre.10-2.2

Otro de los desafíos es mantener los cursos abiertos, ya que conforme avanzan los períodos del año, la deserción toma más fuerza, debido a razones particulares de los y las estudiantes, entre las que destacan cambios de horarios laborales, nuevas oportunidades de trabajo en otros lugares, traslados del lugar de residencia y otras de índole personal. La deserción del estudiantado se vuelve todo un reto, pues al ser este un proyecto autofinanciado, se necesita una cantidad mínima de estudiantes para mantener la apertura y asegurar los compromisos financieros del mismo.

Otro desafío para el proyecto, que tiene cierta relación con el anterior, se refiere a los costos económicos para el proyecto, ya que dependiendo de las particularidades económicas de cada zona se reduce el precio, y se cubren los gastos con algún remanente de dinero de otra sede; pero si la sede base reduce sus entradas de dinero a través de las matrículas, se podría afectar la estabilidad financiera del proyecto, ya que llega un momento en que pareciera que es más lo que asume el proyecto en gastos que las entradas económicas, de ahí que esa parte se debe manejar con mucho cuidado desde la coordinación.

Y el último desafío es la necesidad de mayor recurso humano para el área de coordinación del proyecto, debido a la cantidad de estudiantes que se maneja con la expansión, las labores administrativas se elevan, por lo que se deben doblar los esfuerzos para mantener y cumplir con los requerimientos del proyecto para asegurar el buen funcionamiento y la calidad de sus cursos.

\section{Consideraciones finales}

Este proyecto considera que sí se generan cambios de aprendizaje en la población. Entre los y las estudiantes de este proyecto hay un gran número de jóvenes que salieron del colegio y no pudieron ingresar a una universidad, por lo que este proyecto les brinda una oportunidad para mejorar mientras ingresan a una carrera; otros han encontrado trabajo o mejorado las condiciones de este gracias al mejoramiento del inglés o francés logrado con nuestros cursos. De nuestros estudiantes y egresados hemos recibido distintos testimonios gracias a su participación en este proyecto, como que son considerados los mejores en inglés de sus grupos del colegio y/o escuela gracias a la capacitación adicional que reciben, además manifiestan que pudieron comunicarse con éxito en un país extranjero, ya que aplicaron lo aprendido en nuestras aulas; algunos han obtenido una certificación mediante el DELF en el caso del idioma francés o certificación hasta del C1 en inglés. 
También, gracias a este proyecto, más personas oyentes son capaces de comunicarse con personas sordas por medio de los cursos de Lesco. Es importante mencionar que se han acogido estudiantes con algún tipo de discapacidad, entre ellas síndrome de Down, espina bífida y autismo; para asegurarles calidad en el proceso de enseñanza-aprendizaje se han organizado capacitaciones en torno a estos temas en las que han participado como audiencia docentes de otras instituciones de la comunidad.

Cabe resaltar que este es el único programa de la zona que capacita a niños y niñas en otro idioma desde los tres años de edad, permitiendo que se inicien en el aprendizaje de un nuevo idioma y otros procesos de desarrollo y socialización desde una edad temprana, lo que permite la construcción de actitudes positivas hacia otras lenguas, facilitando su aprendizaje en el futuro.

Sin duda, la enseñanza de idiomas no debe verse únicamente desde el paradigma económico de la globalización, más importante aún es verlo como una manera de transformación social, ya que contribuye a la reducción de estereotipos con respecto a otras comunidades o culturas, favoreciendo de este modo valores como el respeto y la solidaridad, tan necesarios en nuestros días. Con solo esto, ya cumple una misión de agente de cambio. Entonces, el proyecto contribuye, desde su espacio de acción, a la transformación de la sociedad puntarenense, al permitirle acceso a educación de calidad. La educación, claro está, tiene el poder de formar y transformar.

Por último, este proceso de expansión ha sido muy satisfactorio para este proyecto al poder cumplir con las metas de un objetivo específico planteado durante el año 2018, además, logra hacer que la Universidad de Costa Rica cumpla con ese vínculo universidad-sociedad, y prueba de ello son este tipo de proyectos desarrollados desde la acción social; sin duda, es de suma importancia, para que este tipo de proyectos tenga éxito en las comunidades, vincular a diferentes actores sociales para el logro del mismo, como el caso de este proyecto, que involucró a asociaciones de desarrollo, municipalidades y escuelas. No cabe duda de que en este país la necesidad de capacitación en las diferentes regiones es vital para generar oportunidades para su población. 


\section{Referencias bibliográficas}

Acuña, G., Barahona, M. y Ceciliano, Y. (2010). Extensión y dominio efectivo del inglés como segunda lengua en el sistema educativo costarricense: situación actual, problemas y desafios. III Informe del Estado de la Educación, Conare.

Asociación de Estudiantes de la Sede de Occidente (setiembre, 2014). Propuesta para la ejecución de un proceso de regionalización integral. Ponencia presentada en el VII Congreso Universidad de Costa Rica. San José, Costa Rica.

Consejo Nacional de Rectores (1998). Convenio Marco para el Desarrollo de las Sedes Regionales Interuniversitarias en Educación Superior Universitaria Estatal de Costa Rica. Compendio Leyes, Decretos y Convenios de la Educación Superior Universitaria Estatal en Costa Rica. Recuperado de https://www.conare.ac.cr/images/docs/leyes_ acuerdos/OPES_2-2013_Leyes_decretos_convenios.pdf

Hasbún, L. (2007). Algunas razones por las que nuestro mundo debería ser multilingüe. Revista de Lenguas Modernas, 6, 109-117.

López, R. (2014). Puntarenas hacia el camino del multilingüismo: impacto del proyecto de idiomas de la Sede del Pacífico de la Universidad de Costa Rica. Revista InterSedes, 15(30), 132-150.

López, R. (2017). Idiomas para la Comunicación Internacional. Reflexiones a cinco años de su inicio en la capacitación de idiomas en el Pacífico central de Costa Rica. Revista Extensión en Red, (8), e003. https://doi. org/10.24215/18529569e003.

Navarrete, K. (febrero, 2018). Desarrollo Intercultural a través del aprendizaje de idiomas en estudiantes de cursos de extensión universitaria en la Región Pacifico Central Costarricense. Ponencia presentada en el $11^{\circ}$ Congreso Universidad. La Habana, Cuba.

Universidad de Costa Rica (2016). Vicerrectorías. Recuperado de https:// www.ucr.ac.cr/organizacion/vicerrectorias/accion-social.html

Vicerrectoría de Acción Social (2015). ¿Quiénes somos? Recuperado de https://accionsocial.ucr.ac.cr/quienes-somos 